\title{
Editorial
}

\section{Fifth World Veterinary Orthopaedic Congress: An Outstanding Success}

\author{
Kenneth A. Johnson ${ }^{1}$ \\ 1 Department of Orthopaedics, Sydney School of Veterinary Science, \\ University of Sydney, Sydney, New South Wales, Australia \\ Vet Comp Orthop Traumatol 2018;31:v.
}

The fifth WVOC jointly hosted by ESVOT and VOS in Barcelona Spain from 12 to 15 September 2018 was without any doubt, the best one yet. With over 70 internationally acclaimed speakers, dispersed across six concurrent sessions, the 1000 participants were spoilt for choice. A new feature of the congress was the provision of simultaneous translation into French, German, Italian and Spanish. Barcelona was at her best, with perfect weather, an excellent venue and the distractions of an irresistible array of art and culture, especially the work of Gaudi, Picasso, Miro and Dali.

State of the art lectures were very popular. Professor Martin Fischer from Friedrich-Schiller University Jena in Germany revealed new data on canine locomotion gained by biplanar fluoroscopy, such as the rotary motion of the scapula during running, coxofemoral subluxation in hip dysplasia, and lateral motion of the pelvis in the French bulldog. In addition, Dr. Dror Paley, the father of CORA and limb deformity talked about new techniques of minimally invasive

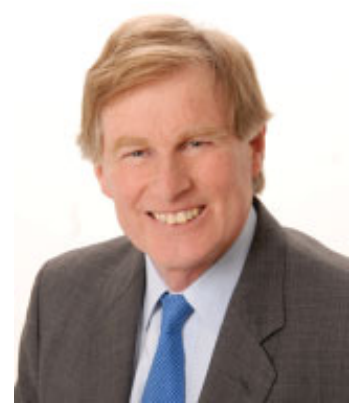

Kenneth A. Johnson limb lengthening using telescoping interlocking nails in the tibia and femur. In comparison to conventional techniques of Ilizarov type distraction, the morbidity of this surgery was minimal. Indeed, there were so many other interesting lectures on new techniques, technology and trends, it is impossible to mention but a few things. One had to be there to appreciate it.

The outstanding social programme provided additional opportunities at which to meet old friends and colleagues, and to make new ones. Congratulations to Professor Peter Böttcher, ESVOT president, and the entire team who worked very hard to make this a memorable event. Thank you so much. You have set a very "high bar" for the organizers of the next WVOC. We are looking forward to it!

(c) 2018 Georg Thieme Verlag KG Stuttgart · New York
DOI https://doi.org/ $10.1055 / \mathrm{s}-0038-1675554$. ISSN 0932-0814. 